
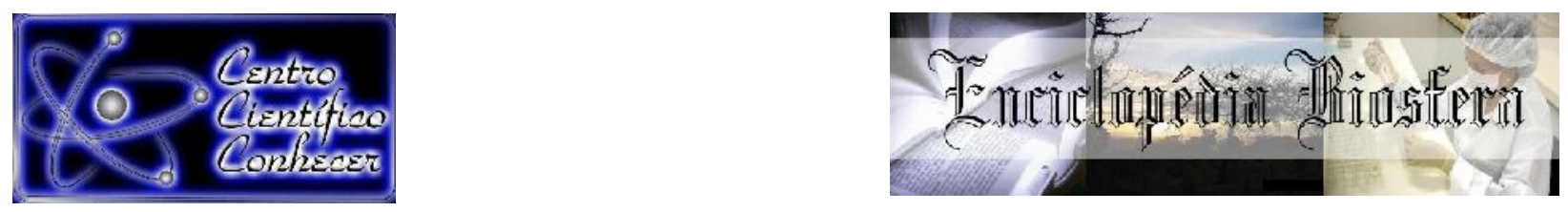

\title{
ANÁLISES ESTATÍSTICAS DO NÚMERO DE CASOS E ÓBITOS NOVOS NO ESTADO PERNAMBUCANO: SUCESSO OU FRACASSO AO COMBATE DA COVID-19?
}

\author{
Maurício Assuero de Lima Freitas ${ }^{1}$, Maria de Fátima da Silva ${ }^{2}$, Elâne Rafaella \\ Cordeiro Nunes Serafim ${ }^{3}$
}

1-Doutor em Economia pela Universidade Federal de Pernambuco; Prof. Adjunto do

Departamento de Ciências Contábeis e Atuariais da UFPE

2-Mestre em Administração e Desenvolvimento Rural pela Universidade Federal

Rural de Pernambuco; Professora da Faculdade de Ciências Sociais dos PalmaresFACIP

3-Doutora em Ciência Animal Tropical pela Universidade Federal Rural de Pernambuco; Analista em vigilância em saúde da Secretaria de Saúde de Lagoa dos Gatos-PE- (elane.rafaella@gmail.com)

Recebido em: 15/02/2021 - Aprovado em: 15/03/2021 - Publicado em: 30/03/2021 DOI: 10.18677/EnciBio_2021A2

\begin{abstract}
RESUMO
A pandemia por COVID-19 afetou o comportamento humano e econômico no mundo inteiro. Medidas de isolamento social, lockdown, limitação de circulação de pessoas em supermercados e bancos, impuseram uma nova ordem de relacionamento humano. Em todo mundo, o rastro de mortes é intenso e a falta de vacina e de conhecimento sobre o número de assintomáticos geram uma incerteza muito grande quando se inicia o processo de volta à normalidade. Este trabalho faz uma avaliação da pandemia no Estado de Pernambuco utilizando dados, entre 05 de março e 30 de abril de 2020, do Ministério da Saúde e da Secretaria de Planejamento e Gestão do Estado, fez-se análise de estatística descritiva e distribuição de probabilidade. $O$ trabalho analisa os casos e os óbitos por faixa etária, local de residência do paciente e gênero. Usando a quantidade de internações em 2019 como proxi para averiguar o possível impacto das comorbidades de risco, observou-se que não há argumentos para se aceitar que essa faixa de risco tem sido atingida. Um segundo resultado do trabalho sugere que a doença deixou de ser aleatória porque os casos diários estão violando as previsões. Isso sugere que o Estado está falhando no combate à doença.
\end{abstract}

PALAVRAS-CHAVE: coronavírus; pandemia; síndrome respiratória aguda grave;

\section{STATISTICAL ANALYSIS OF THE NUMBER OF NEW CASES AND DEATHS IN THE STATE OF PERNAMBUCO: SUCCESS OR FAILURE IN COMBATING COVID-19?}

\section{ABSTRACT}

The COVID-19 pandemic has affected human and economic behavior worldwide. Measures of social isolation, lockdown, limiting the movement of people in supermarkets and banks, imposed a new order of human relationship. All over the world, the trail of deaths and intensities and the lack of vaccine and lack of 
knowledge about the number of asymptomatic individuals generate a great deal of uncertainty when the process of returning to normality begins. This work makes an assessment of the pandemic in the state of Pernambuco using data, between March 05 and April 30 2020, from the Ministry of Health and the State Department of Planning and Management, an analysis of descriptive statistics and probability distribution was made. The work analyzes the cases and deaths by age group, place of residence of the patient and gender. Using the number of hospitalizations in 2019 as a proxy to ascertain the possible impact of risk comorbidities, it was observed that there are no arguments to accept that this risk range has been reached. A second result from the work suggests that the disease is no longer random because daily cases are violating predictions. This suggests that the state is failing to fight the disease.

KEYWORDS: coronavirus; pandemic; severe acute respiratory syndrome

\section{INTRODUÇÃO}

A família do coronavírus é conhecida desde 1960 e é responsável por infecções respiratórias como a Síndrome Respiratória Aguda Grave (SARS) e a Síndrome Respiratória do Oriente Médio (MERS) e no entendimento de Boffa et al. (2020) a sua origem pode estar relacionada a uma mutação no coronavírus que infecta morcegos, quebrando a barreira genética para se adaptar a uma nova espécie. Primeiramente a COVID-19 apresenta-se clinicamente, como uma síndrome semelhante à gripe e vai evoluindo para uma doença respiratória com presença de febre persistente.

O período de incubação desse novo coronavírus varia de dois a 14 dias, seu alcance geográfico, o número de infectados e a taxa de mortalidade têm deixado a população em estado de insegurança e medo (ORNELL et al., 2020). Quanto à disseminação, Medeiros (2020) afirma que uma pessoa infectada pode transmitir o vírus para duas a quatro pessoas, denotando o alto potencial de contágio e 0 aumento exponencial da incidência dos casos.

$\mathrm{Na}$ pandemia causada pelo novo Coronavírus (COVID-19), até o dia 30 de abril de 2020, o Brasil tinha registrado 85.380 casos novos de pessoas infectadas pelo novo coronavírus, com 5.901 óbitos ocorridos, ou seja, uma taxa de letalidade de $6,91 \%$. Comparativamente a outros países, o Brasil registrava 28,01 óbitos por milhão de habitantes, nível muito inferior ao que se observou na Bélgica, Itália, Espanha, França (BRASIL, 2020).

O Estado de São Paulo detinha $33,61 \%$ dos casos com letalidade de 8,28\%, no entanto, quando se avaliava o número de óbitos por milhão de habitantes, 0 Estado do Amazonas liderava com 102,54, seguido por Pernambuco com 59,12 óbitos por milhão de habitantes; o Ceará com 52,78 óbitos por milhão de habitantes e o Rio de Janeiro com 51,72 óbitos por milhão de habitantes. O Estado de São Paulo apresentava 49,46 óbitos por milhão de habitantes. $O$ desastre da pandemia no Brasil foi intensificado pelas ocorrências desses cinco Estados (BRASIL, 2020).

O Estado de Pernambuco, foco do interesse desse estudo, registrou na região metropolitana da capital, composta por 15 municípios, a maior concentração de casos novos e de óbitos (PERNAMBUCO, 2020a). De acordo com o Decreto № 5278 de 2020, o Estado dispunha de 140 leitos clínicos e 75 de Unidade de Tratamento Intensivo (UTI), mas com o aumento dos casos de COVID-19, este número foi ampliado para 1443 leitos clínicos e 657 leitos de UTI (PERNAMBUCO, 2020 b), ou seja, esse total de leitos representava $13,36 \%$ da quantidade de leitos destinados ao Sistema Único de Saúde (SUS), que é de 15.560 (BRASIL, 2020c). O 
Estado teve 20.961 leitos para internação, dos quais $74,71 \%$ eram vinculados ao SUS.

Pernambuco registrou como paciente zero um casal de idosos, com idades de 66 e 71 anos, recém chegados da Itália. A primeira morte por COVID-19 no Estado aconteceu em 25 de março de 2020. A vítima, do sexo masculino, 85 anos de idade, apresentava histórico de diabetes, hipertensão, além de cardiopatia isquêmica. Com 30 dias dos primeiros casos confirmados, o Estado contabilizava 960 casos de pacientes com o novo coronavírus e 85 mortes (PERNAMBUCO, 2020a).

Para frear a pandemia, o Governo do Estado adotou o isolamento social, a exemplo de outros estados, com o consequente fechamento das atividades econômicas e educacionais, restringindo serviços não essenciais, proibindo aglomerações, fechando praias, shoppings, templos religiosos, dentre outros. Além disso, a Secretaria de Saúde Estadual em parceira com as secretarias municipais de saúde elaboraram planos de contingências e normas sanitárias a serem adotadas pela população e serviços de saúde (PERNAMBUCO, 2000a). No entanto, mesmo diante dessas medidas os números de novos casos da COVID-19 e mortes permaneceram crescendo, indicando que as medidas protocolares adotadas não eram suficientes para frear o avanço da doença (INLOCO, 2020).

Nesse contexto, este trabalho teve por objetivo analisar o comportamento do número de casos e óbitos, por COVID-19, no Estado de Pernambuco.

\section{MATERIAL E METÓDOS}

Buscou-se pelos métodos de estatística descritiva e inferencial descrita no estudo de Silva (2016). Assim a população da pesquisa compreendeu o Estado Pernambucano. O critério de seleção foi o número de notificações, óbitos e casos novos da COVID-19 no Estado.

Os dados foram coletados de forma indireta, por meio de consulta às seguintes bases de dados: SIH (Sistema de Informações Hospitalares), SIA (Sistema de Informações Ambulatoriais) e SIM (Sistema de Informações de Mortalidade), disponibilizados pelo Departamento de Informática do Sistema Único de Saúde (DATASUS), no endereço eletrônico (http://www2.datasus.gov.br).

Além das bases de dados Secretaria de Planejamento e Gestão do Estado de Pernambuco (https://dados.seplag.pe.gov.br/apps/corona.html) e do Centro de informações Estratégias de Vigilância em Saúde de Pernambuco (https://www.cievspe.com/). As variáveis analisadas foram caso novo e óbito por COVID-19, no período entre 05 de março e 30 e abril de 2020.

Após a coleta dos dados, utilizou-se estatística descritiva para cálculo das medidas de tendência central como média, mediana e cálculo de medidas de variação como variância e desvio padrão. A hipótese de normalidade foi analisada com os testes de Doornik-Hansen, Shapiro-Wilk W, Lilliefors e Jarque-Bera, utilizando o software Greatl, considerando como dados a quantidade de dias decorridos entre a notificação e o óbito e os casos novos versus óbitos, com intervalo de confiança de $95 \%$ e erro padrão de $5 \%$.

\section{RESULTADOS E DISCUSSÃO}

Pernambuco, até 30 de abril de 2020 registrou em 131 municípios do estado 6.876 casos da COVID-19, fato que representa um incremento de 33 municípios com novos casos, comparativamente a 13 de abril quando havia casos em 98 municípios do estado. A letalidade do Estado foi de $8,22 \%$.

A figura 1 mostra que existiu uma tendência crescente de casos novos, no 
entanto, não de forma exponencial. Em relação aos óbitos, o comportamento foi mais estacionário. A média diária de casos novos foi de 137,52, com desvio padrão de 173,53 , o que denota uma variabilidade de $126,18 \%$. O valor máximo de casos novos, 682, ocorreu no dia 30 de abril, o que foi um indício de preocupação por sugerir tendência de alta. O intervalo $[88,20 ; 186,84]$, contém a verdadeira média de casos novos, com $95 \%$ de confiança.

FIGURA 1. Distribuição temporal dos casos novos e óbitos novos de COVID-19, em Pernambuco no período de 05 de março a 30 de abril de 2020.

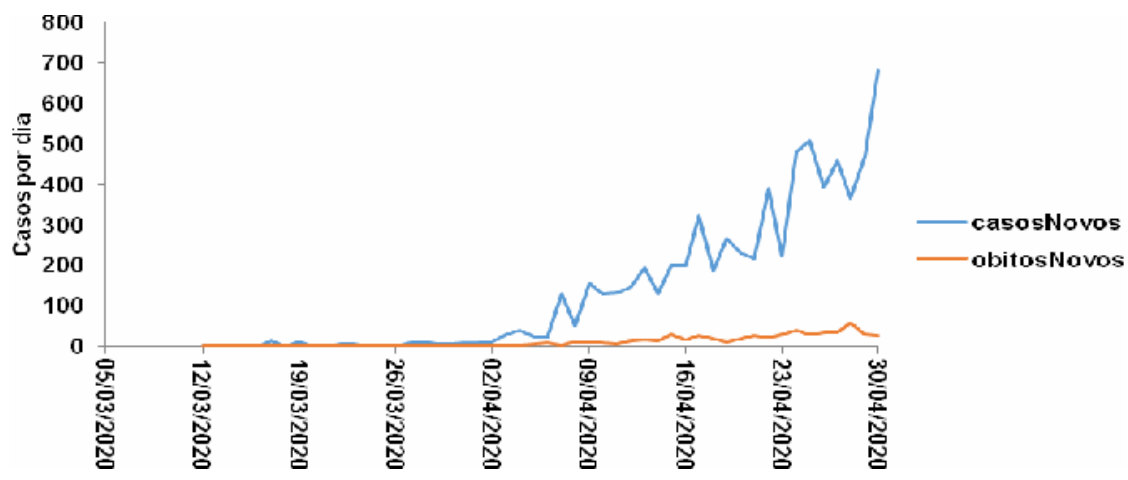

Fonte: Os autores, (2020).

A média de óbitos diários foi de 11,30 , com desvio padrão de 13,72 , ou seja, uma variabilidade de $121,42 \%$. Note-se que a variabilidade dos óbitos foi menor do que a de casos novos. Isso pode ser um indício que corrobore a intensidade do contágio e justifique as medidas de restrição. A verdadeira média diária de óbitos estava no intervalo $[7,40 ; 15,20]$ com $95 \%$ de confiança.

As medidas adotadas em Pernambuco, de acordo com os dados do presente estudo, comparando a variabilidade dos óbitos e de casos novos, indicaram que produziu efeito importante no avanço da epidemia, no entanto, não na proporção esperada, pois os óbitos continuaram em crescimento. Esse contraste pode estar relacionado com a taxa de isolamento social no Estado. Quando as primeiras medidas foram adotadas, a taxa de isolamento social, de acordo com INLOCO (2020) estava abaixo de $45 \%$, com o avançar das restrições das medidas, como exemplo, fechamento temporário de escolas e de serviços não essenciais, essa taxa alcançou patamar máximo de $62 \%$ de isolamento. No entanto, esse patamar foi reduzindo com o avançar do tempo, seja pela a não adesão da população e pelo aumento de fluxo de pessoas devido à flexibilização de algumas atividades industriais e comerciais no Estado, como o ramo de confecção na região Agreste do Estado que retornou as atividades para produção de Equipamentos de Proteção Individual, e consequentemente houve o retorno de funcionários as fábricas e ao comércio de aviamento.

Ressalta-se que às medidas de controle adotadas foram norteadas por estudos científicos, como o realizado por Hui e Zumla (2020) na China, que citam que é essencial ocorrer à implantação de procedimentos rigorosos de controle de infecções com precauções respiratórias e de contato. As consequências dessas medidas foram identificadas como possível impacto econômico e social na população devido ao tempo desse distanciamento, o baixo crescimento econômico, alto desemprego (ORNELL; SCHUCH, 2020), e o aumento das despesas 
governamentais, concomitantemente a queda na arrecadação, em um conjunto de ocorrências que fez reduzir a renda, aumentar a pobreza e intensificar desigualdades, afetando, substancialmente, o bem estar social. Ademais, medidas aprovadas no congresso geraram transferências aos Estados e municípios e a suspensão da dívida dos entes federados com a União através da Lei complementar 149/19 (BRASIL, 2020).

Além desse aspecto de adoção de medidas profiláticas, é importante mencionar que as pessoas continuaram apresentando patologias e agravos comuns, e a depender da complexidade houve um fluxo de atendimento de pessoas que residem no interior do Estado deslocarem-se para as macrorregiões de saúde (Recife, Caruaru, Petrolina e Serra Talhada), conforme a tabela 1, tornando-as vulneráveis a se infectarem e propagarem o vírus no interior do Estado.

TABELA 1. Distribuição do número de pacientes internados em hospitais no mês de março de 2020 em Pernambuco, por microrregião de saúde de residência do paciente e macrorregião de internação do mesmo.

\begin{tabular}{lrrrr}
\hline \multirow{1}{*}{$\begin{array}{c}\text { Microrregião de Saúde de } \\
\text { residência }\end{array}$} & \multicolumn{3}{c}{ Macrorregião de saúde de internação de } \\
& Recife & Caruaru & Petrolina & Serra Talhada \\
\cline { 2 - 5 } & 20471 & 22 & 1 & 3 \\
\hline I Microrregião - Recife & 3841 & 77 & 0 & 0 \\
II Microrregião - Limoeiro & 1953 & 32 & 0 & 0 \\
III Microrregião - Palmares & 2076 & 2926 & 0 & 0 \\
IV Microrregião - Caruaru & 740 & 1535 & 6 & 0 \\
V Microrregião - Garanhuns & 473 & 150 & 208 & 0 \\
VI Microrregião - Arcoverde & 121 & 2 & 4 & 595 \\
VII Microrregião - Salgueiro & 107 & 1 & 46 & 66 \\
VIII Microrregião - Petrolina & 124 & 8 & 1 & 1152 \\
IX Microrregião - Ouricuri & 222 & 50 & 0 & 0 \\
X Microrregião - Afogado da & 205 & 36 & 37 & 32 \\
Ingazeira & 30333 & 4839 & 303 & 1848 \\
XI Microrregião - Serra Talhada & & &
\end{tabular}

Fonte: Brasil, (2020c).

Vale salientar que muito pacientes internados ou em atendimento em Pernambuco apresentam doença considerada fator de risco para COVID-19, como hipertensão arterial sistêmica e diabetes mellitus (BRASIL, 2020c). Nesse contexto, ao avaliar o risco do Estado de Pernambuco face às doenças consideradas fatores de risco para COVID-19, considerando como referência o ano de 2019, conforme apresentado na tabela 2, verificou-se que pacientes com hipertensão arterial sistêmica, diabetes mellitus, cardiopatia, doença cardiovascular crônica, obesidade, doença renal, neoplasia, Síndrome de Fournier e asma já apresentam maior risco de internamento no Estado. Evidenciando, que pacientes infectados pelo coronavírus que apresentam comorbidades são propensos ao risco, mas, pode não se configurar a causa primária de tantas mortes no Estado. 
TABELA 2: Resumo Estatístico das Comorbidades por milhão de habitantes

\begin{tabular}{lrrrrr}
\hline \multicolumn{1}{c}{ Medidas } & Tuberculose & Neoplasias & Cardíacas & Obesidade & Diabetes \\
\hline Média & 418,94 & 3768,42 & 3810,74 & 45,52 & 691,27 \\
Erro padrão & 37,18 & 273,54 & 261,88 & 10,00 & 47,86 \\
Mediana & 396,33 & 3306,26 & 3616,62 & 26,23 & 661,00 \\
Desvio padrão & 193,21 & 1421,33 & 1360,79 & 51,98 & 248,68 \\
Mínimo & 146,87 & 1804,52 & 1940,33 & 0,00 & 322,80 \\
Máximo & 955,46 & 6721,21 & 7674,95 & 174,63 & 1454,66 \\
\hline
\end{tabular}

Fonte: Os autores, (2020).

Em relação a variável: casos novos, apresentou distribuição normal ${ }^{1}$ e com base nisso, a figura 2 mostra conjuntamente a frequência acumulada de casos novos por dia e a previsão pela distribuição normal.

FIGURA 2. Relação da previsão de casos novos pela distribuição normal.

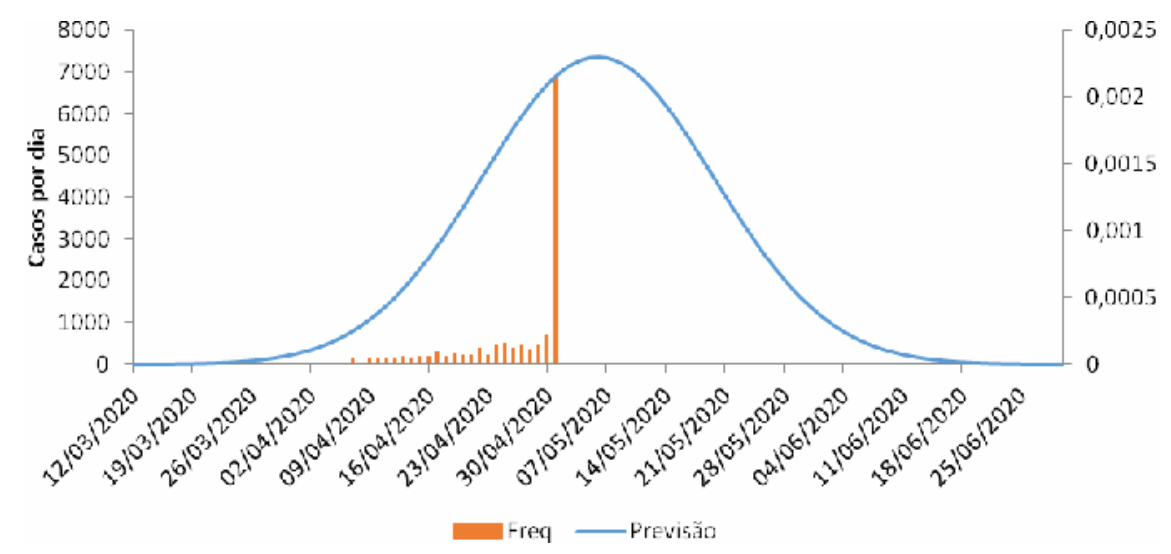

Fonte: os autores, (2020).

Notou-se que a distribuição de frequência estava à esquerda da média da previsão, sugerindo que o pico de contágio seria 06.05.2020. Entre as datas 09 de abril e 02 de junho teve-se 95\% dos casos, ou seja, após 02 de junho esperava-se a redução dos casos novos.

Os dados da SEPLAG não possuíam informação completa e por isso fez-se uma análise local considerando uma amostra com 5.614 observações. Nesse universo, $55,02 \%$, ou 3.089 , acometeram mulheres. Cerca de $90,25 \%$ ocorreram nas cidades da RMR - Região Metropolitana do Recife.

Dos 2.525 homens contaminados, $89,18 \%$ residem na RMR, assim como $91,23 \%$ das mulheres. Dez cidades da RMR apresentaram $88,85 \%$ dos casos, sendo a cidade do Recife a que teve maior preponderância, com $51,72 \%$ dos casos de todo estado e $57,13 \%$ dos casos da região metropolitana. Do total de casos, $3,87 \%$ estavam internados em leito de UTI e $23,37 \%$ foram curados (recuperados).

A concentração de casos em Recife pode estar relacionada às características de mobilidade populacional da região. Sabe-se que a RMR é composta por uma população de 3.690 .547 milhões de habitantes, e o volume de deslocamento por

\footnotetext{
${ }^{1}$ O software Greatl teste a hipótese de normalidade pelos teste de Doornik-Hansen, Shapiro-Milk,Lilliefors e Jarque-Bera e em todos eles aceita-se a hipótese de normalidade dos dados.
} 
motivo de trabalho entre os 14 municípios que compõe a RMR registrado é de 448.912 pessoas. O deslocamento maior foi para o município de Recife, 307.202 indivíduos, procedentes em sua maioria de Jaboatão dos Guararapes (108.029), seguido por Olinda (74.328), Paulista (50.033), Camaragibe (28.235) e São Lourenço da Mata (13.920). Além disso, 46.703 pessoas deixaram a metrópole diariamente a capital para realizar suas atividades em outros municípios, sendo preferencialmente para Jaboatão dos Guararapes (17.528) e Olinda (11.079) (SILVA; QUEIROS, 2017). Ou seja, a mobilidade diária de pessoas em Recife é um meio facilitador da disseminação do vírus nessa região, já que além do deslocamento, uma parcela da população utiliza transportes públicos nesse deslocamento. Como o vírus pode permanecer viável em algumas superfícies por 72 horas o risco de propagação intensificou-se e consequentemente houve maior concentração de casos nessa localidade.

Além dessa mobilidade rotineira na RMR é importante destacar que em fevereiro de 2020, duas semanas antes do registro do primeiro caso de COVID-19 em Pernambuco, ocorreu o carnaval. Esse evento é destaque na cidade do Recife, marcado pela multiplicidade de ritmos, públicos e espaços, e pela convivência entre a tradição e o novo. Essa estrutura do carnaval do Recife propicia aos foliões que estes transitem entre os polos, participando de diferentes espaços da festa (GAIO; LEÃO, 2013). O carnaval intensificou o fluxo de pessoas no município e pode ter sido outro fator facilitador da disseminação do vírus nessa localidade.

Em relação à análise dos óbitos por COVID-19 registrado em Pernambuco, determinou-se a quantidade de dias decorridas entre a notificação e o óbito. Essa variável apresentou média de 9,84 dias com desvio padrão de 5,99 dias, portanto, o intervalo $[9,25 ; 10,43]$ contém a verdadeira média de dias entre notificação e óbito. 0 maior valor observado nessa variável foi 30 dias e a moda da distribuição foi 0 , ou seja, houveram 12 casos nos quais o óbito ocorreu no dia da notificação. Registre-se que, em 200 casos a quantidade de dias entre óbito e notificação foi negativa, ou seja, a notificação ocorreu após o óbito. Essa notificação após o óbito pode estar relacionada à escassez de testes diagnósticos na época, o que dificultou o diagnóstico precoce de casos positivos. De acordo com Brasil (2020a) caso a colheita de material biológico não tenha sido realizada no paciente em vida, deve-se proceder à coleta post-mortem no serviço de saúde, por meio de swab na cavidade nasal e de orofaringe, para posterior investigação pela equipe de vigilância local.

Ressalta-se que o aumento da detecção de casos novos de COVID-19 associa-se a disponibilização de testes de diagnóstico que começaram a ser distribuídos para os Estados com maior intensidade a partir da segunda semana de Abril de 2020, e consequentemente houve um acréscimo na quantidade de casos detectados. De acordo com o COSEMS-PE (2020) o Estado de Pernambuco recebeu 8.867 kits de Teste Rápido para detecção da COVID-19. Deste quantitativo, a partir do dia 07 de abril de 2020, a Secretaria Estadual de Saúde de Pernambuco (SES-PE) reteve 2.626 Kits, e o restante, 6.241 Kits foram distribuídos entre os 184 municípios para serem usados em funcionários da saúde, segurança pública, idosos sintomáticos. Após essa distribuição o registro de casos por COVID-19 tendeu a aumentar no Estado devido à ampliação do diagnóstico de casos suspeitos.

Essa situação evidenciou que os testes sorológicos se destacaram na pandemia como uma ferramenta essencial para rastrear a propagação da doença. Inicialmente eram em um número reduzido, mas com a avançar do tempo houve um aumento na variedade de testes disponíveis comercialmente (PATEL et al., 2020)

Observa-se na figura 3 que a maior incidência dos óbitos ocorreu na faixa 
etária acima de 40 anos, registrando $85,12 \%$ dos óbitos. O maior percentual de óbitos $(25,06 \%)$ ocorreu na faixa entre 60 e 69 anos. Do total de óbitos masculinos, 184 , houve $84,40 \%$ na faixa etária acima de 50 anos. Para as mulheres, $74,01 \%$ dos óbitos ocorreram na faixa etária acima de 60 anos. Esses achados assemelham-se a literatura, onde pesquisas anteriores citam que a idade é um fator de risco para COVID-19, assim como presença de doenças concomitantes e presença de infecção secundária (RUAN et al., 2020).

FIGURA 3. Distribuição dos óbitos por COVID-19 por faixa etária

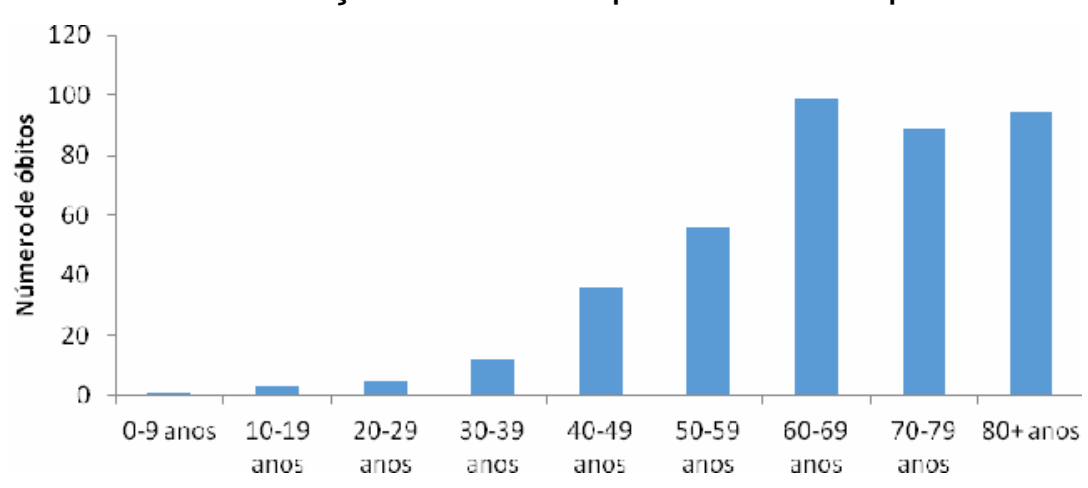

Fonte: os autores, (2020).

Tendo em vista que a população de cada cidade varia, uma forma de equalizar a representativamente do número de óbitos é mensurando por unidade de cem mil habitantes (cmh). A tabela 3 mostra essa distribuição apenas para cidades com mais de 100 mil habitantes.

TABELA 3: Relação da distribuição do óbitos por cem mil habitantes, para municípios pernambucanos com mais de 100 mil habitantes,

\begin{tabular}{l|r|r|r}
\hline Município & População & \multicolumn{1}{|c|}{ Óbitos } & \multicolumn{1}{c}{ Óbitos/cmh } \\
\hline São Lourenço da Mata & 113.230 & 31 & 27,38 \\
\hline Recife & 1.645 .727 & 298 & 18,11 \\
\hline Olinda & 392.482 & 58 & 14,78 \\
\hline Camaragibe & 157.828 & 22 & 13,94 \\
\hline Jaboatão dos Guararapes & 702.298 & 81 & 11,53 \\
\hline Vitória de santo antão & 138.757 & 15 & 10,81 \\
\hline Paulista & 331.774 & 34 & 10,25 \\
\hline Cabo de Santo Agostinho & 207.048 & 18 & 8,69 \\
\hline Igarassu & 117.019 & 10 & 8,55 \\
\hline Garanhuns & 139.788 & 3 & 2,15 \\
\hline Caruaru & 361.118 & 7 & 1,94 \\
\hline
\end{tabular}

Fonte: Os autores, (2020).

A cidade de São Lourenço da Mata apresentou o maior número de óbitos por cem mil habitantes. Esse quantitativo chegou a ser 1,5 vezes maior do que a cidade do Recife, 14 vezes maior do que se observou em Caruaru, a maior cidade da região Agreste do Estado. É importante mencionar, que estudos iniciais sobre a COVID-19 realizado por Hui e Zumla (2020) na China citam que é essencial ocorrer a implantação de procedimentos rigorosos de controle de infecções com precauções respiratórias e de contato. 
Em Pernambuco, o governo estadual regulamentou medidas temporárias para enfrentamento da emergência de saúde pública, como isolamento social, fechamento atividades educacionais, econômicas e de lazer. Obviamente, outras consequências, além da econômica, advirão dessas medidas, uma das quais, registrada por Garcia e Duarte (2020), tratou da vulnerabilidade de mulheres e crianças à violência doméstica e intrafamiliar.

Essa taxa de mortalidade em São Lourenço da Mata evidenciou o quanto a enfermidade vem sendo letal nesse município, sendo necessária que haja uma adequação e intensificação das medidas sanitárias adotadas visando reduzir essa situação. Além da ampliação na detecção precoce de casos de pessoas com COVID-19 e uma assistência de saúde adequada dessas pessoas. Um fator que pode ter favorecido essa circulação intensa do vírus foi a mobilidade de residentes desse município na RMR, principalmente em Recife, que é um dos locais com taxas elevadas de casos positivos. De acordo com Silva e Queiros (2017), diariamente 11.962 pessoas residentes em São Lourenço da Mata deslocam-se para Recife para realizar atividades econômicas, trabalhar ou estudar.

Diante desse contexto apresentado, observou-se que como ainda o uso de qualquer medicação não tem comprovação cientifica dos efeitos, a indicação mais eficiente é o isolamento social (MEDEIROS, 2020), associado à vacina que vem sendo apresentadas por diferentes laboratórios. A questão da higiene, do uso de máscaras, do guardar distância em filas, etc são meios adicionais para evitar a propagação do vírus. Embora Oliveira e Pinto (2020) afirmem que as medidas restritivas que limitam a circulação dos indivíduos e isolamento social são medidas necessárias, o número de óbitos crescente mostra que estas não são suficientes.

\section{CONCLUSÃO}

O Estado de Pernambuco vivenciou, em diversos aspectos tais como idade, sexo e local de residência, uma situação grave no enfrentamento da pandemia de COVID-19 na qual os casos deixaram de ser aleatórios.

Os dados de abril de 2020 sinalizaram uma tendência da intensidade do mal no estado e transcorridos quase um ano, observa-se que os danos reais são superiores aos previstos.

Esse trabalho mostrou que a violação da aleatoriedade teve como consequência erros de estimativas por parte de diversos centros de pesquisa. Indubitavelmente, as ações desenvolvidas no estado não se mostraram coerentes a abrangência da pandemia.

Por tanto, evidencia-se que houve falhas no combate à doença e indica-se a necessidade de avaliar a previsibilidade de estabilização dos casos novos ao adotar medidas estratégias de prevenção. Assim como, buscar agregar ao mesmo tempo diferentes mecanismos profiláticos como o isolamento social e adoção de bloqueios mais objetivos para os primeiros dias de sintomas da doença.

\section{REFERÊNCIAS}

BOFFA, J.; MHLABA, T.; SULIS, G.; MOYO, S.; SIFUMBA, Z.; PAI, M.; DAFTARY, A. COVID-19 and tuberculosis in South Africa: A dangerous combination. South African Medical Journal, v. 110, n. 5, p: 1-2, 2020. Disponível em < https://pesquisa.bvsalud.org/controlecancer/resource/pt/mdl-32657710>

BRASIL. Lei complementar 149/19. Estabelece o Programa de Acompanhamento e Transparência Fiscal, o Plano de Promoção do Equilíbrio Fiscal, altera a Lei 
Complementar oㅜ 101, de 4 de maio de 2000, a Lei Complementar oㅜ 156, de 28 de dezembro de 2016, a Lei no 12.348 , de 15 de dezembro de 2010, a Lei oㅜ 12.649, de 17 de maio de 2012 e a Medida Provisória no 2.185-35, de 24 de agosto de 2001, Brasília, DF. Disponível em: <https://www.camara.leg.br/proposicoesWeb/prop_mostrarintegra;jsessionid=BEFB3 2236574B344246CB5918D024154. proposicoesWebExterno1? codteor $=1762548 \&$ file name=PLP+149/2019 $>$. Acesso em: 12 abr de 2020.

BRASIL. Ministério da Saúde. Coronavírus: o que você precisa saber e como prevenir ou impedir. 2020. Brasil, 2020a. Disponível em: <https://saude.gov.br/saude-de-az/coronavírus>. Acesso em: 12 abr 2020.

BRASIL. Ministério da Saúde. Manejo de corpos no contexto do novo Coronavírus COVID-19. Brasília/DF: Ministério da Saúde. 1a edição, versão 1, 2020b. p 17. Disponível em: < https://www.gov.br/saude/ptbr/media/pdf/2020/dezembro/15-1/af_manejo-corpos-covid_2ed_27nov20_isbn.pdf>.

BRASIL, Ministério da Saúde. Banco de dados do Sistema Único de SaúdeDATASUS. Disponível em: <http://www.datasus.gov.br> Acesso em 3 mar 2020c.

COSEMS-PE. Conselho de Secretarias Municipais de Saúde de Pernambuco. (2020. 07 de maio). Proposta de distribuição de Testes Rápidos - COVID-19, Terceira Entrega - 07/05/2020. Disponível em: <https://www.cosemspe.org/wpcontent/uploads/2020/05/PROPOSTA-TESTE-COVID-

19_COSEMS_08.05.2020.pdf>. Acesso em: 20 abr. 2020.

GAIÃO, B. F. S.; LEÃO, A. L. M. S. Muitas festas numa só: a configuração do campo do carnaval do Recife. Organizações \& Sociedade, v. 20, n. 64, p:131-144, 2013. Disponível em: < http://dx.doi.org/10.1590/S1984-92302013000100009>.

GARCIA, L. P.; DUARTE, E. Intervenções não farmacológicas para o enfrentamento à epidemia da COVID-19 no Brasil. Revista Epidemiologia e Serviço em Saúde, v. 29, n.2, p: 2020-222, 2020. Disponível em: <http://dx.doi.org/10.5123/s167949742020000200009>.

HUI, D.; ZUMLA, A. Severe Acute Respiratory Syndrome: Historical, Epidemiologic, and Clinical Features. Infectious disease clinics of North America, v. 33, n.4, p:869-889, 2019. Disponível em: < https://pesquisa.bvsalud.org/portal//resource/\%20es/mdl-31668196>.

INLOCO. Mapa Brasileiro da COVID-19, 2020. Disponível em: < https://mapabrasileirodacovid.inloco.com.br/pt/>. Acesso em: 24 abr 2020.

PERNAMBUCO. Lei no 13.979, de 6 de fevereiro de 2020. Dispõe sobre as medidas para enfrentamento da emergência de saúde pública de importância internacional decorrente do coronavírus responsável pelo surto de 2019. Disponível em: < http://www.in.gov.br/en/web/dou/-/lei-n-13.979-de-6-de-fevereiro-de-2020242078735>. Acesso em: 20 abr 2020a

PERNAMBUCO. Resolução CIB/PE №. 5278, de 02 de Abril de 2020. Aprovar O Plano de Contingência para infecção pelo Coronavírus (COVID-19) com Leitos 
de Enfermaria e Leitos de Terapia Intensiva (Anexo I) do Estado de Pernambuco. Recife, 4 de abril de 2020 Diário Oficial do Estado de Pernambuco Poder Executivo Ano XCVII - NÀ 63 - 17. Disponível em: $<$ http://web.transparencia.pe.gov.br/ckan/dataset/legislacao-COVID-19.>. Acesso em: 20 abr 2020b.

MEDEIROS, E. A. S. Challenges in the fight against the COVID-19 pandemic in university hospitals. Revista Paulista de Pediatria, v. 38, n. 1, p:2020-086, 2020. Disponível em: < https://doi.org/10.1590/1984-0462/2020/38/2020086>. Acesso em: 20 abr 2020.

OLIVEIRA, G. M. M.; PINTO, F. J. COVID-19: A Matter Close to the Heart. International Journal of Cardiovascular Sciences, Epub 17, 2020. Disponível em: <https://doi.org/10.36660/ijcs.20200057 >. Acesso em: 20 abr 2020.

ORNELL, F.; SCHUCH, J. B.; SORDI, A. O.; KESSLER, F. H. P. "Pandemic fear" and COVID-19: mental health burden and strategies. Revista Brasileira de Psiquiatria [on-line], Epub 03, 2020. Disponível em: < https://doi.org/10.1590/15164446-2020-0008>. Acesso em: 22 abr 2020.

PATEL, R.; BABADY, E.; THEEL, E. S.; STORCH, G. A.; PINSKY, B. A.; et al.,; Report from the American Society for Microbiology COVID-19 International Summit, 23 March 2020: Value of Diagnostic Testing for SARS-CoV-2/COVID-19. American Society for Microbiology v. 11, n.2, p:e00722-20, 2020. Disponível em: < DOI: 10.1128/mBio.00722-20>. Acesso em: 22 abr 2020.

RUAN, Q.; YANG, K.; WANG, W.; JIANG, L.; CANÇÃO J. Clinical predictors of mortality due to COVID-19 based on an analysis of data of 150 patients from Wuhan, China. Cuidados Intensivos Medico, v. 46, n.5, P: 846-848, 2020. Disponível em: < doi: 10.1007/s00134-020-05991-x.>. Acesso em: 22 abr 2020.

SILVA, J.G.; QUEIROZ, S.N.. Mobilidade Pendular na Região Metropolitana de Recife (RMR). In: Encontro Nacional da Associação Nacional de PósGraduação e Pesquisa em Planejamento Urbano e Regional. Organizadores: Camila D'Ottaviano, Eduardo Nobre. - São Paulo: Faculdade de Arquitetura e Urbanismo da Universidade de São Paulo, 2017. 337 p. Disponível em: http://anpur.org.br/xviienanpur/principal/publicacoes/XVII.ENANPUR_Caderno_de_R esumos/XVII.ENANPUR_CadernodeResumoDigital.pdf>. Acesso em: 21 abr 2020.

SILVA, P. M. Metodologia estatística aplicada na análise da violência escolar: apuração e interpretação de dados na rede pública do estado de Goiás. Revista online de Política e Gestão Educacional, v. 20, n.2, p:322-336, 2016.Disponível em: <DOI:http://dx.doi.org/10.22633/rpge.v20.n2.9478>. Acesso em: 22 abr 2020. 